\title{
Left Atrioventricular Artery
}

National Cancer Institute

\section{Source}

National Cancer Institute. Left Atrioventricular Artery. NCI Thesaurus. Code C116177.

The first posterolateral branch originating from the posterior atrioventricular left circumflex artery in left dominant and mixed circulations. 